\title{
Attenuation of gentamicin ototoxicity by glutathione in the guinea pig in vivo ${ }^{1}$
}

\author{
Susan L. Garetz, Richard A. Altschuler, Jochen Schacht * \\ Kresge Hearing Research Institute, Department of Otolaryngology, Unitersity of Michigan, 1301 East Ann Street, Ann Arbor, MI 48109-0506. USA
}

(Received 10 November 1993; Revision received 3 March 1994; Accepted 16 March 1994)

\begin{abstract}
The effect of glutathione co-therapy on the expression of gentamicin ototoxicity was tested in pigmented guinea pigs. The first group of animals was injected with gentamicin $(100 \mathrm{mg} / \mathrm{kg}$ body weight/day) for two weeks followed by 10 weeks of rest. A second group received glutathione by gastric gavage immediately prior to each gentamicin injection. Two groups of controls were treated either with saline injections or glutathione gavage alone. Auditory brainstem responses, taken at 2 -week intervals, revealed a progressive gentamicin-induced hearing loss reaching a 30 to $40 \mathrm{~dB}$ threshold shift at $2 \mathrm{kHz}$, approximately $60 \mathrm{~dB}$ at 8 $\mathrm{kHz}$ and $80 \mathrm{~dB}$ at $18 \mathrm{kHz}$. Glutathione co-therapy slowed the progression of hearing loss and significantly attenuated the final threshold shifts by 20 to $40 \mathrm{~dB}$. Morphological evaluation confirmed hair cell loss after gentamicin treatment and protection by glutathione. Drug serum levels were assayed at 2 and 7 days of treatment. There were no differences between the gentamicin (mean $=183 \mu \mathrm{g} / \mathrm{ml}$; range, 90 to 300 ) and the gentamicin/glutathione group (mean $=164 \mu \mathrm{g} / \mathrm{ml} ; \mathrm{range}, 80$ to 320 ). Antimicrobial activity of gentamicin was tested against Staphylococcus aureus and Pseudomonas aeruginosa. A 30-fold molar excess of glutathione did not influence the efficacy of gentamicin. These studies suggest that glutathione protects against ototoxicity by interfering with the cytotoxic mechanism.
\end{abstract}

Key words: Aminoglycosides; Ototoxicity; Protection; Glutathione; Free radicals; Scavengers

\section{Introduction}

After having been in therapeutic use for nearly five decades, aminoglycoside antibiotics are currently receiving renewed attention. They were the first efficacious drugs to combat tuberculosis, and opportunistic infections associated with AIDS have now brought a worldwide resurgence of this disease (Shafer et al., 1991). Despite improvements in therapeutic regimen and development of new aminoglycosides, the nephroand ototoxic potentials associated with this group of drugs have not yet been eliminated. The incidence of ototoxicity in patients - in the form of vestibular disturbances or auditory impairments - can be as high as $18 \%$ for gentamicin and as low as $2 \%$ for netilmicin

\footnotetext{
* Corresponding author. Fax: (313) 764-0014.

${ }^{1}$ A preliminary report of this research has appeared in Abstract form. Garetz, Rhee and Schacht, Abstr. Assoc. Res. Otolaryngol. 15, 110, (1993).
}

(Fec, 1980; Matz, 1986). The damage frequently begins soon after the initiation of drug treatment, may continue after cessation of therapy and is mostly permanent (Hawkins, 1976).

Numerous attempts have been made to reduce the severity and frequency of the side-effects of aminoglycoside antibiotics, but these have been uniformly unsuccessful or unconvincing (reviewed by Federspil, 1979). A major handicap in designing rational preventive measures has been the lack of understanding of the precise mechanisms leading to hair cell destruction. However, recent studies have demonstrated the existence of a cytotoxic gentamicin metabolite ('gentatoxin') providing a new theoretical basis for such attempts (Huang and Schacht, 1990; Crann et al., 1992). In fact, the cytotoxic actions of this metabolite against outer hair cells in vitro can be prevented by antioxidants and sulfhydryl compounds (Garetz and Schacht, 1992; Garetz et al., 1994).

Glutathione $(\mathrm{GSH})$ is a thiol-containing tripeptide found in virtually all mammalian cells. It plays a key 
role in a number of cellular processes including detoxification of xenobiotics and protection against reactive oxygen species (Jakoby, 1978; Listowsky et al., 1988; Meister, 1991). Depletion of GSH potentiates drug-induced ototoxicity in vivo (Hoffman et al., 1988), and its presence protects outer hair cells in vitro against cytotoxicity of the gentamicin metabolite (Garetz and Schacht, 1992; Garetz et al., 1994). These findings prompted the current study with the goal to determine if GSH could also protect the mammalian auditory system in vivo.

\section{Materials and Methods}

\section{Experimental groups}

Pigmented adult guinea pigs (Murphy, Plainfield, $\mathrm{NJ}$ ) weighing $250-400 \mathrm{~g}$ were used in this study. Control groups (saline or GSH only) contained 5 animals each. Groups receiving gentamicin initially consisted of 12-13 animals to allow for a sufficient number of surviving animals given the known morbidity of the drug at high doses. Each group was treated daily for 14 days. Group 1 was injected with gentamicin sulfate (100 $\mathrm{mg}$ gentamicin base $/ \mathrm{kg}$ body weight) intramuscularly. Group 2 was anesthetized with metaphane (drop inhalation method) and given $0.6 \mathrm{ml}$ of $0.3 \mathrm{M} \mathrm{GSH}$ via gastric gavage. Thirty min later gentamicin was injected as above. Group 3 was anesthetized as above and then given $0.6 \mathrm{ml}$ glutathione via gastric gavage. Group 4 was injected intramuscularly with saline in a volume equivalent to that received by animals in group 1 . Only animals who survived for the entire study period were included in the analysis. These were 5 of 5 animals in the saline group; 7 of 13 receiving gentamicin; 4 of 5 receiving glutathione; and 6 of 12 on combined gentamicin/glutathione treatment.

\section{Evoked potentials}

Auditory thresholds were measured for each animal prior to the start of the study and then again at 2, 4, 8 and 12 weeks after beginning of treatment. Animals were anesthetized with $40 \mathrm{mg} / \mathrm{kg}$ ketamine and 10 $\mathrm{mg} / \mathrm{kg}$ xylazine given by intramuscular injection. The recording electrodes consisted of needles inserted subcutaneously below the ipsilateral right pinna (reference electrode) and at the vertex (active electrode). The ground electrode was located in the right thigh because the more conventional use of the opposite ear was precluded by the positioning of the animals for the insertion of earphones. Placed at maximum distance from major vessels, this electrode did not cause any significant heart beat artifacts. Tone bursts of $3 \mathrm{~ms}$ duration with $1 \mathrm{~ms}$ rise and fall times were presented at 2,8 , and $18 \mathrm{kHz}$ to the right ear through an inset speculum in the external auditory meatus. Stimuli were generated by a Fordham oscillator (model AG-298) and delivered via a Beyer DT-48 transducer. ABRs were recorded with a gain of 100,000 and filter settings of $100 \mathrm{~Hz}$ to $3 \mathrm{kHz}$. Average response tracings from 512 sweeps were obtained at $5 \mathrm{~dB}$ intervals near threshold. Thresholds at each frequency were verified twice and defined as the intensity that produced at detectable change from a non-stimulus condition. Individual threshold shifts for each time point were calculated by comparing intensity at threshold to the pretreatment threshold for that animal at the same frequency.

\section{Serum gentamicin levels}

Two hundred $\mu$ l of serum were obtained by toe nail clipping from animals treated with either gentamicin alone or with gentamicin and GSH on treatment days 2 and 7. Samples were taken $30 \mathrm{~min}$ after injection. Cells were removed by centrifugation and plasma was stored at $-20^{\circ} \mathrm{C}$ until analysis. Immediately prior to assay the serum was thawed and diluted $1: 100$ with normal saline. Gentamicin levels were measured using a commercial fluorescence polarization immunoassay (Abbott Diagnostics, Abbott Park, IL).

\section{Cochlear morphology}

An apical opening was made in the extirpated right cochleae which were perfused with $0.1 \mathrm{M}$ sodium cacodylate, $2 \%$ paraformaldehyde, $2 \%$ glutaraldehyde and $2 \mathrm{mM}$ calcium chloride. After $24 \mathrm{~h}$ of gentle rotation in the same fixative at $4^{\circ} \mathrm{C}$, cochleae were rinsed in phosphate buffer at $4^{\circ} \mathrm{C}$ for 2 days. Excess bone was removed and the cochleae were then decalcified in $3 \%$ EDTA at $4^{\circ} \mathrm{C}$ with gentle rotation for $2-3$ weeks. They were then dehydrated through a graded series of alcohol and embedded in EMbed 812 resin. Six-mm sections were cut in a para-modiolar plane and every third mid-modiolar section was mounted on a glass slide and stained with Richardson's stain. Two animals from each of the four treatment condition were processed under double-blind conditions. Six comparable mid-modiolar sections from each animal were assessed using bright-field and differential interference contrast optics on a Leitz photomicroscope.

\section{Antimicrobial activity}

Efficacy of gentamicin alone and in the presence of GSH was tested against Staphylococcus aureus (ATCC 25923) and Pseudomonas aeruginosa (ATCC 27853) in a standard microbiological assay. Twenty $\mu$ l of GSH were dispensed onto a $6 \mathrm{~mm}$ disk containing $10 \mu \mathrm{g}$ of gentamicin. The disks were placed on the surface of a $150 \mathrm{~mm}$ culture plate containing Mueller-Hinton agar at a depth of $4 \mathrm{~mm}$. The surface of the agar had previously been inoculated with a standardized concentration of either strain of bacteria. The inoculated 
plates were incubated overnight at $35^{\circ} \mathrm{C}$ in room air. The diameter of the inhibition zones was measured to the nearest $\mathrm{mm}$ across each disk. These analyses were carried out by the Clinical Microbiology Laboratory, Department of Pathology, University of Michigan.

\section{Results}

\section{Auditory brain stem response}

Auditory thresholds were comparable in all groups at the beginning of the study. Animals injected with saline or gavaged with GSH maintained essentially stable hearing at all of the measured frequencies (Fig. 1). Animals receiving gentamicin showed the expected progressive hearing loss. Consistent with the established pattern of aminoglycoside ototoxicity (Hawkins, 1976; Garetz and Schacht, 1994), the rate of threshold shift and its magnitude increased from low to high frequencies. Also, hearing loss continued after the 2-week treatment and leveled off after 4 weeks at the high frequencies. Threshold shifts ranged from $30 \mathrm{~dB}$ at $2 \mathrm{kHz}$ to about $80 \mathrm{~dB}$ at $18 \mathrm{kHz}$. Animals gavaged with GSH prior to gentamicin injection showed a slower progression of hearing loss at all frequencies and a significant reduction of the final threshold shift. Attenuation of gentamicin toxicity ranged from about $20 \mathrm{~dB}$ at $2 \mathrm{kHz}$ to $40 \mathrm{~dB}$ at $18 \mathrm{kHz}$. The brief anesthesia preceding GSH gavage apparently did not confound the treatment. Under a modified experimental design, glutathione administered without anesthesia also attenuated aminoglycoside-induced hearing loss in animals maintained on a restricted diet (Lautermann and Schacht, 1994).

It should be noted that the group of control animals tested at 12 weeks showed a jump of 30 to $40 \mathrm{~dB}$ in their thresholds. This phenomenon most likely represented a testing artifact since the morphology in those animals (see below) was normal. Since there was no concomitant shift in the treated groups, this potential artifact produced thresholds in the control animals almost identical to those in the gentamicin/GSH group. Because of the uncertainty of the validity of the control thresholds and the resulting bias in interpretation, the 12-week data were not included in the evaluation.

\section{Morphology}

Cochlcac from two animals in each of the four treatment groups were examined histologically. Their morphology supported the electrophysiological findings that treatment with GSH exerted a protective influence on hair cells (Fig. 2). Sections from control animals treated with saline or GSH-gavage only showed normal morphology in all turns (Fig. 2a and b). Guinea pigs treated with gentamicin alone revealed almost complete loss of outer hair cells in the first and second

\section{$2 \mathrm{KHz}$}

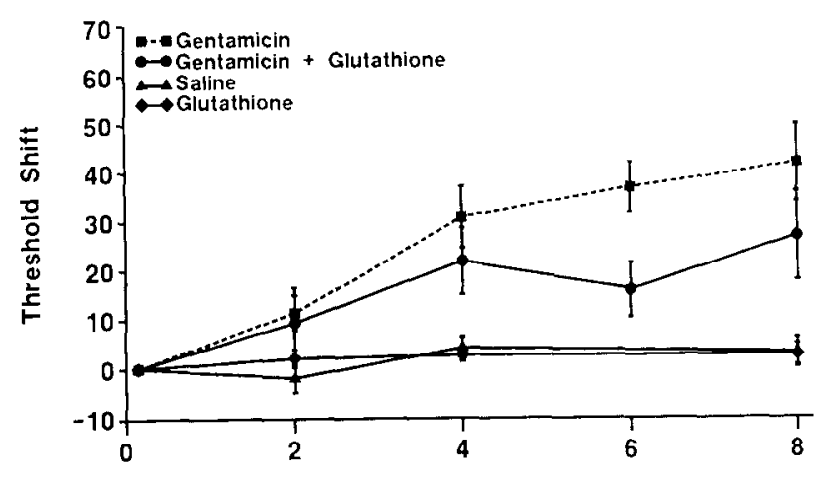

$8 \mathrm{KHz}$

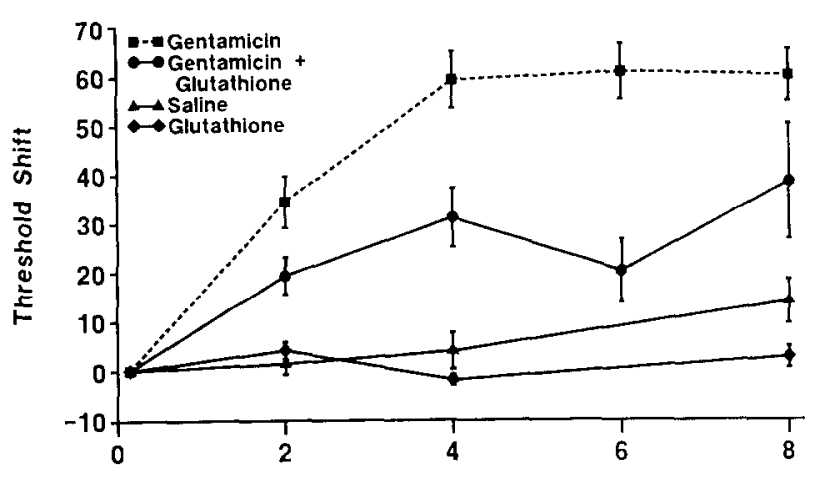

$18 \mathrm{KHz}$

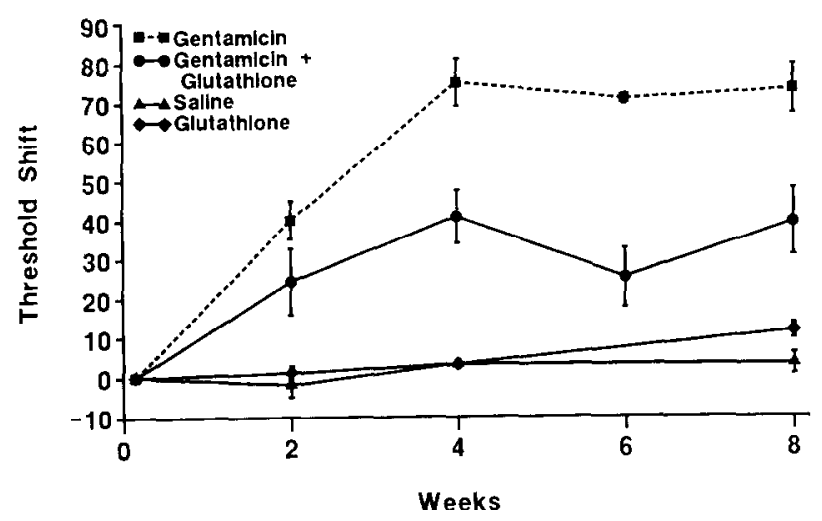

Fig. 1. Time course of threshold shifts at 2,8 and $18 \mathrm{kHz}$. Four groups of animals were treated and ABR thresholds measured as described in 'Methods'. Control groups not receiving gentamicin maintained stable thresholds while gentamicin induced progressive hearing luss. Differences between treatments were evaluated by ANOVA. There were no statistically significant differences between treatment with saline and GSH alone at any frequency. Threshold shifts induced by gentamicin were significantly different $(P<0.05)$ at 8 and $12 \mathrm{kHz}$ beginning at 4 weeks. GSH co-therapy significantly $(P<0.05)$ attenuated gentamicin-induced threshold shifts at 8 and $18 \mathrm{kHz} . N=5$ animals (saline); $N=4$ (GSH only); $N=7$ (gentamicin only); $N=6$ (gentamicin plus GSH). 
turns (Fig. $2 \mathrm{c}$ and d) consistent with the documented pattern of aminoglycoside-induced pathology (Hawkins, 1976; Garetz and Schacht, 1994). There was also some loss of inner hair cells in the first turn. In contrast, less hair cell loss was apparent in animals treated with GSH gavage prior to administration of gentamicin (Fig. $2 \mathrm{e}$ and $\mathrm{f}$.

\section{Serum levels of gentamicin}

Serum levels of gentamicin were unaffected by $\mathrm{GSH}$ treatment. On day 2 and 7, gentamicin concentrations averaged $183 \mu \mathrm{g} / \mathrm{ml}$ (range, 80 to 320 ). This was not statistically different from the mean of $164 \mu \mathrm{g} / \mathrm{ml}$ (range, 90 to 300 ) in the animals gavaged with GSH prior to their gentamicin injections (Fig. 3).
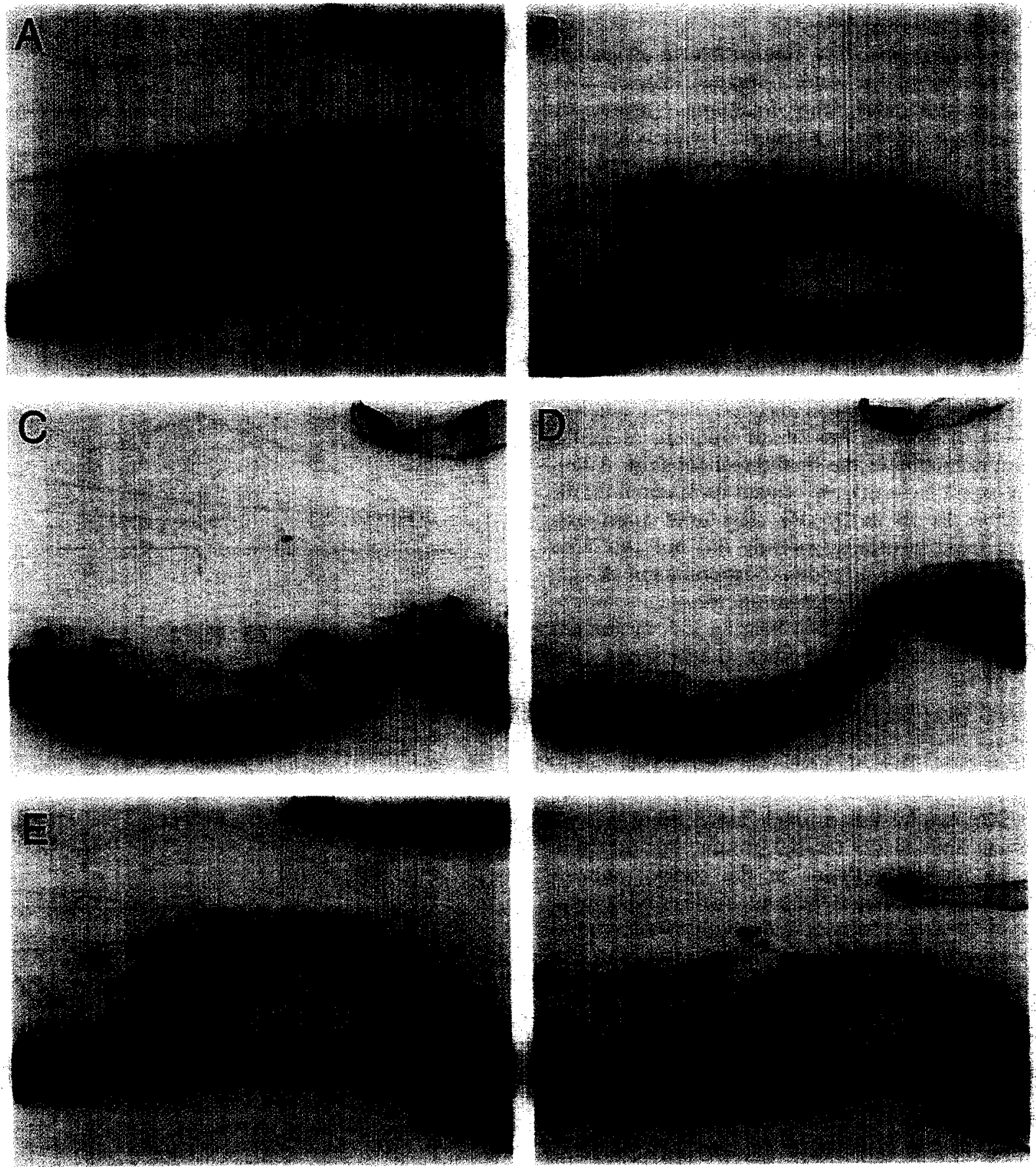

Fig. 2. Histopathology of the organ of Corti. Animals were treated and histological sections obtained from two animals in each group as described in 'Methods'. Representative photomicrographs of mid-modiolar sections through the first turn of the cochlea of guinea pigs treated with saline (A), GSH only(B), gentamicin (C, D) and gentamicin plus GSH (E, F). Hair cell loss is seen with gentamicin (C, D) administration, but less so if there was pre-treatment with GSH before gentamicin (E, F). Saline (A) and GSH (B) controls showed no effects on cochlear structures. 
Table 1

Glutathione does not affect antimicrobial activity of gentamicin

\begin{tabular}{lll}
\hline Condition & \multicolumn{2}{l}{ Inhibition zones } \\
\cline { 2 - 3 } & S aureus & Paeruginosa \\
\hline 20 nmol $(10 \mu \mathrm{g})$ gentamicin & $23 \pm 1$ & $19 \pm 1$ \\
Gentamicin plus 20 nmol GSH & 22 & 19 \\
Gentamicin plus $600 \mathrm{nmol}$ GSH & 22 & 19 \\
\hline
\end{tabular}

Inhibition zones were measured in a microbiological assay against Staphylococcus aureus and Psetudomonas aenuginosa as described in 'Methods'.

\section{Antimicrobial efficacy}

Gentamicin efficacy against Streptococcus aureus and Pseudomonas aeruginosa was tested in a standard antimicrobial assay. The inhibition zones produced by 10 $\mu \mathrm{g}$ gentamicin remained unaffected by GSH up to a 30 -fold molar excess, the highest concentration tested (Table 1).

\section{Discussion}

Enteral administration of glutathione prior to gentamicin treatment clearly reduced the extent of gentamicin-induced hearing loss in guinea pigs at high and low frequencies as compared to animals receiving gentamicin alone. The effect was already evident at the end of the two-week regimen, the earliest time at which responses were measured. In agreement with the established pattern of gentamicin toxicity, the hearing loss progressed after cessation of treatment but the attenuating effect of GSH was also maintained. The electrophysiological observations were confirmed by the histology: gentamicin produced the expected loss of hair cells while GSH co-therapy attenuated hair cell death.

Depletion of GSH, either by starvation (Prazma et al., 1983; Hoffman et al., 1987) or by enzymatic inhibition (Hoffman et al., 1988) has been shown to potentiate the ototoxicity associated with aminoglycosides administered either alone or in conjunction with loop diuretics. Our data is the first indication that the converse effect also occurs. Importantly, the afforded protection is not mediated by an effect on drug serum levels which otherwise would compromise the therapeutic efficacy of the treatment. The best explanation, in view of results recently obtained by our laboratory (Crann et al., 1992; Garetz and Schacht, 1992; Garetz et al., 1994) is a direct interference by GSH with the cytotoxic mechanism of gentamicin. In vitro toxicity assays have demonstrated that gentamicin will not destroy outer hair cells in short-term culture. However, after an enzymatic reaction the resulting gentamicin metabolite is acutely cytotoxic. This metabolic step, apparently necessary for the expression of gentamicin toxicity, can be inhibited in vitro by radical scavengers and sulfhydryl compounds including GSH (Garetz et al., 1994). As demonstrated here, the toxic effect of gentamicin in vivo can likewise be attenuated by GSH.

Sulfhydryl compounds as ameliorating agents for ototoxic drugs have been tested before with varying success. One example of the difficulties encountered in establishing the efficacy of antidotes was the experience with the sulfhydryl agent 2,3-mercaptopropanol. Claims of attenuation of aminoglycoside ototoxicity by

\section{Serum Gentamicin Levels}
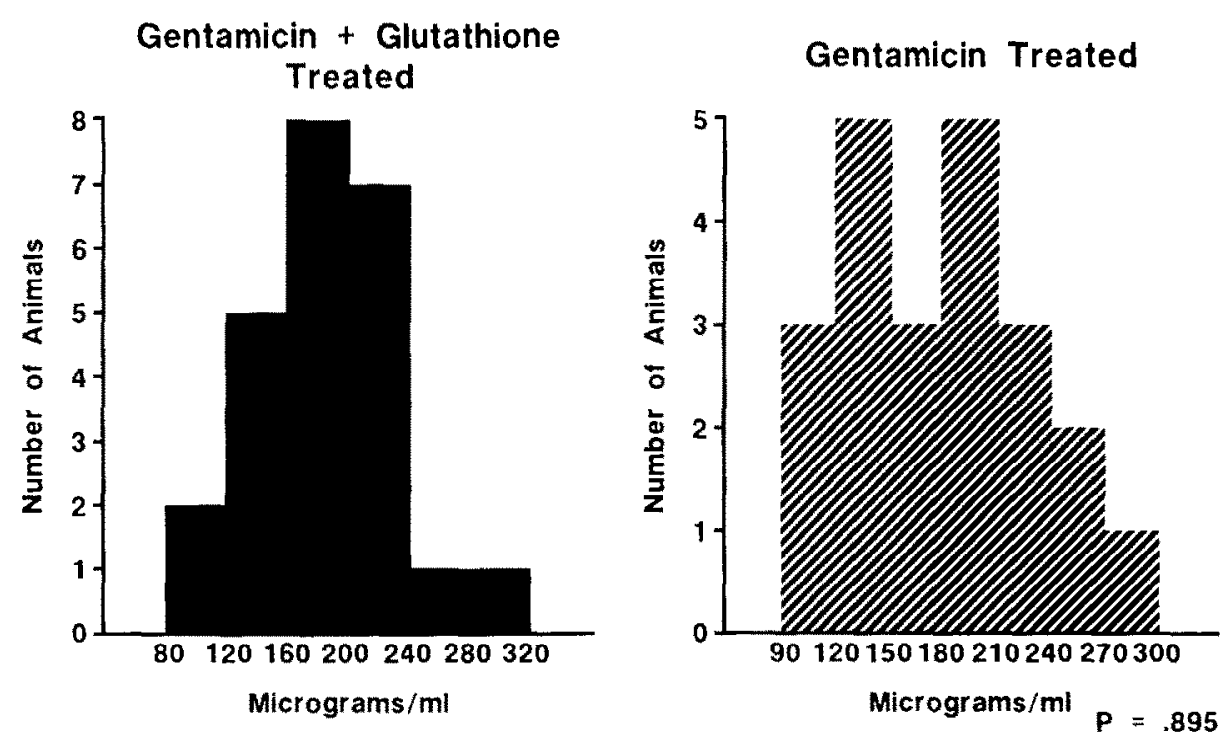

Fig. 3. Serum levels of gentamicin. Serum levels of gentamicin were obtained after 2 and 7 days of treatment as described in 'Methods'. Values in animals receiving GSH co-therapy did not differ statistically from values in animals receiving gentamicin alone. 
this compound had been made in the $1950 \mathrm{~s}$ and $1960 \mathrm{~s}$ based on animal experimentation and clinical trials. Subsequently, these reports were disputed and 2,3mercaptopropanol was shown to be toxic in itself (Federspil, 1979). GSH was also tested and found ineffective against kanamycin (Darrouzet, 1967). It should be considered, however, that GSH homeostasis in vivo is subject to many factors including nutritional status, stress, and health of the animal. Successful amelioration of toxic side effects may therefore depend on the physiological state of the animal. An animal already challenged by undernutrition or pathogenic infection should be more susceptible to ototoxic insults than normal animals because of weakened intrinsic protective mechanisms. Thus, such animals may respond more favorably to co-therapy. This variable might explain the scattered claims of success with vitamins, amino acids or similar supplements. Interestingly, after the completion of this study it became known that the colony from which the animals were derived suffered from a viral infection. Although impossible to determine in retrospect, these animals may have provided a test model already challenged by infections. This aspect of restoring protective homeostatic mechanisms by GSH warrants further exploration.

Co-therapy introduces a second drug and therefore possible added complications. However, sulfhydryl compounds are well-established drugs in antioxidant therapy (Rice-Evans and Diplock, 1993). In particular, GSH can be manipulated by diet or intravenous administration (Hagen et al., 1990; Wendel and Jaeschke, 1981) without apparent side effects of elevated serum levels. On the contrary, GSH administration has been investigated in humans to prevent adverse side effects of a variety of medications (Cavalletti at al., 1986; Oriana et al., 1987).

Finally, an effective intervention against gentamicin ototoxicity must not compromise the therapeutic efficacy of the drug. GSH appears to fulfill this condition. Firstly, it does not alter serum levels of gentamicin. Secondly, it does not affect the bactericidal efficacy of the antibiotic as determined against two representative pathogens. Thus, the lack of deleterious actions and the ease of administration suggest GSH as an ideal agent to reduce the ototoxicity associated with aminoglycoside administration.

\section{Acknowledgments}

We wish thank Dr. Sanford Bledsoe for help with the electrophysiological recordings. We also like to acknowledge William Hubbard and the Clinical Microbiology Laboratory at the University of Michigan for the determination of the antimicrobial efficacy of gen- tamicin. This work was supported by grant DC-00124 and training grant DC- 00024 from the National Institutes of Health.

\section{References}

Cavalletti, F., Tofanetti, O. and Zunino, F. (1986) Comparison of reduced glutathione with 2-mercaptoethane sulfonate to prevent cyclophosphamide-induced urotoxicity. Cancer Lett. 32, 1-6.

Crann, S.A., Huang, M.Y., McLaren, J.D. and Schacht, J. (1992) Formation of a toxic metabolite from gentamicin by a hepatic cytosolic fraction. Biochem. Pharmacol. 43, 1835-1839.

Darrouzet, J. (1967) Essais de protection de l'organe de Corti contre l'ototoxicité des antibiotiques. Rev. Laryngol. 3-4, 187-203.

Federspil, P. (1979) Antibiotikaschäden des Ohres. Barth, Leipzig, pp. 86-89.

Fee, W.E. jr. (1980) Aminoglycoside ototoxicity in the human Laryngoscope Suppl 24, 1-19.

Garetz, S. and Schacht, J. (1992) Sulfhydryl compounds reduce gentamicin-induced outer hair cell damage in vitro. Abs. Assoc. Res. Otolaryngol. 15, 110.

Garetz, S., Rhee, D.J. and Schacht, J. (1993) Attenuation of gentamicin ototoxicity by glutathione. Abs. Assoc. Res. Otolaryngol. 16, 141.

Garetz, S.L., Rhee, D.J. and Schacht, J. (1994) Sulfhydryl compounds and antioxidants inhibit cytotoxicity to outer hair cells of a gentamicin metabolite in vitro. Hear. Res., in press.

Garetz, S. and Schacht, J. (1994) Ototoxicity: Of mice and men. In: Handbook of Auditory Research (Fay, R.R. and Popper, A.N., Eds.), Vol. VI: Clinical Aspects of Hearing (VanDeWater, T.R. Ed.), Springer-Verlag, New York, in press.

Hagen, T.M., Wierzbicka, G.T., Bowman, B.B. AW, T.Y. and Jones, D.P. (1990) Bioavailability of dietary glutathione: effect on plasma concentration. Am. J. Physiol. 259, G524-G529.

Hawkins, J.E. jr. (1976) Drug ototoxicity. In: Handbook of Sensory Physiology (W.D. Keidel and W.D. Neff, Eds.), Springer, New York, Vol. 5, pp. 707-748.

Hoffman, D.W., Whitworth, C.A., Jones, K.L. and Rybak, L.P. (1987) Nutritional status, glutathione levels, and ototoxicity of loop diuretics and aminoglycoside antibiotics. Hear. Res. 31. $217-222$.

Hoffman, D.W., Whitworth, C.A., Jones-King, K.L. and Rybak, L.P. (1988) Potentiation of ototoxicity by glutathione depletion. Ann. Otol. Rhinol. Laryngol. 97, 36-41.

Huang, M.Y. and Schacht, J. (1990) Formation of a cytotoxic metabolite from gentamicin by liver. Biochem. Pharmacol. 40 R11-R14.

Jakoby, W.B. (1978) The glutathione S-transferases: a group of multi-functional detoxification proteins. Adv. Enzymol. 46, 383414.

Lautermann, J. and Schacht, J. Effects of glutathione and antioxidants on gentamicin ototoxicity in different nutritional states. Abstr. Assoc. Res. Otolaryngol. 17, 64, 1994.

Listowsky, I., Abramovitz, M., Homma, H. and Niitu, Y. (1988) Intracellular binding and transport of hormones and xenobiotics by glutathione S-transferases. Drug Met. Rev. 19, 305-318.

Matz, G.J. (1986) Amminoglycoside ototoxicity. Am. J. Otolaryngol. 7, 117-119.

Meister, A. (1991) Glutathione deficiency produced by inhibition of its synthesis, and its reversal; application in research and therapy. Pharmac. Ther. 55, 155-194.

Oriana, S., Bohm, S., Spatti, G., Zunino, F. and Di Re, F. (1987) A preliminary clinical experience with reduced glutathione as protector against cisplatin-toxicity. Tumori 73, 337-340. 
Prazma, J., Garrison, H.G., Williford, S.K., Ferguson, S.D., Fisher, J., Drake, A. and Klingler, L.E (1983) Alteration of aminoglycoside antibiotic ototoxicity: effect of semistarvation. Ann. Otol. Rhinol. Laryngol. 92, 178-182.

Rice-Evans, C.A. and Diplock, A.T. (1993) Current status of antioxidant therapy. Free Radical Biol Med 15, 77-96.

Shafer, R.W., Chirgwin, K.D., Glatt, A.E., Dahdouh, M.A., Landesman, S.H. and Suster, B. (1991) HIV prevalence, immunosup- pression, and drug resistance in patients with tuberculosis in an area endemic for AIDS. AIDS 5, 399-405.

Wendel, A. and Cikryt, P. (1980) The level and half-life of glutathione in human plasma. FEBS Lett. 120, 209-211.

Wendel, A. and Jaeschke, H. (1981) Drug-induced lipid peroxidation in mice. III. Glutathione content of liver, kidney, and spleen after intravenous administration of free and liposomally entrapped glutathione. Biochem. Pharmacol. 31, 3607-3611. 